\title{
Apprendre à inhiber : une pédagogie innovante au service des apprentissages scolaires fondamentaux (mathématiques et orthographe) chez des élèves de 6 à 11 ans
}

\author{
https://doi.org/10.24046/neuroed.20120101.55
}

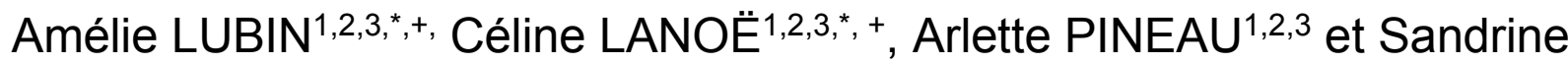 ROSSI $I^{1,2,3}$}

\author{
${ }^{1}$ CNRS, unité 3521, LaPsyDÉ, Paris, France \\ 2 Université Paris Descartes, Sorbonne Paris Cité, unité 3521, LaPsyDÉ, Paris, France \\ 3 Université de Caen Basse-Normandie, unité 3521, LaPsyDÉ, Caen, France \\ + Ces auteurs ont contribué équitablement à cette recherche. \\ *Courriels : amelie.lubin@parisdescartes.fr et celine.lanoe@unicaen.fr
}

\begin{abstract}
Résumé
Certaines situations scolaires sont source de conflit cognitif entre deux savoirs ; des connaissances nouvelles interférant avec des connaissances anciennes. Pour surmonter ce conflit, l'élève doit inhiber la stratégie apprise antérieurement, devenue inefficace, au bénéfice d'une nouvelle stratégie pertinente. Certains blocages, erreurs ou difficultés des élèves nous ont incitées à transférer au monde de la classe des outils centrés sur l'apprentissage à l'inhibition, déjà éprouvés en laboratoire à l'aide de techniques comportementales et d'imagerie cérébrale. Ce projet a pris forme dans le cadre d'un partenariat scientifique entre l'Inspection Académique du Calvados (France) et le Laboratoire de Psychologie du Développement et de l'Éducation de l'enfant (LaPsyDÉ). Nous proposons une démarche pédagogique innovante d'apprentissage à l'inhibition réalisée en classe dans deux domaines scolaires différents (mathématiques et orthographe) avec des élèves de 6 à 11 ans. En partant d'erreurs récurrentes observées par les enseignants chez leurs élèves, nous avons élaboré un dispositif didactique expérimental d'apprentissage à l'inhibition dont nous avons testé l'efficacité. Les résultats de ces deux études mettent en évidence l'avantage d'un apprentissage à l'inhibition par rapport à un apprentissage classique. Ainsi, sensibiliser le terrain éducatif à l'importance du contrôle cognitif dans les apprentissages est fondamental pour permettre aux élèves d'apprendre à mieux apprendre.
\end{abstract}




\section{Introduction}

«Louis a 4 dizaines. Léa a 35 unités. Qui a le plus d'éléments ?»

« Léa! » répondra un élève de 7 ans, car « 35 est plus grand que 4 ».

« Je les manges » écrira un élève de 10 ans lors d'une dictée, car « après

les, je mets un $\mathrm{s} »$.

Quel enseignant ne s'est pas arraché les cheveux face à ce type de réponses dans de telles situations-problèmes ? Sommes-nous ici face à un défaut de compréhension (la notion scolaire n'étant pas acquise), ou pouvons-nous reconsidérer ce type d'erreur à la lumière d'un autre point de vue, celui d'un défaut d'inhibition? En mathématiques, dans l'exemple ci-dessus, la bonne réponse consiste à comparer les nombres, après les avoir transformés dans la même unité de valeur, mais souvent les élèves ne parviennent pas à inhiber l'automatisme de comparaison directe des nombres. II en est de même en orthographe, car fréquemment, les élèves d'école élémentaire commettent l'erreur " je les manges ». Ce n'est pas qu'ils ignorent la règle selon laquelle " avec je, le verbe s'accorde au singulier », mais ils éprouvent des difficultés à inhiber l'automatisme " après les, je mets un $s$ ». La tentation est sans doute ici trop grande, en raison de la proximité du terme « les » avec le verbe dans la phrase. Ces deux exemples illustrent des situations scolaires de conflit cognitif entre deux savoirs, entre deux règles en compétition.

La pédagogie de l'inhibition s'avère alors essentielle afin d'éviter à l'élève de tomber dans les pièges qu'il rencontre à l'école. En effet, il ne suffit pas de connaître les règles, il faut en permanence inhiber nos automatismes. Or, est-il possible de développer à l'école une pédagogie de l'inhibition et du contrôle cognitif ? Certains auteurs ont déjà entrepris cette démarche dans le cadre du programme "Tools of the Mind" avec des enfants d'âge préscolaire (Diamond, 2007, 2011) ou encore dans le programme d'entraînement et de développement des compétences cognitives avec des enfants d'âge scolaire (Gagné et Ainsley, 2002; Gagné et Longpré, 2004; Gagné et Noreau, 2005). Ces derniers visent à entrainer de façon transversale les fonctions exécutives dans des situations de la vie scolaire qui ne sont pas ciblées par un domaine d'apprentissage particulier. Notre approche métacognitive s'inscrit dans ce courant, car elle vise à enseigner aux élèves une méthodologie de travail centrée sur le contrôle cognitif et la détection de conflit. Toutefois, contrairement aux programmes précédents, elle se focalise sur l'apprentissage d'une notion dans le cadre d'une séquence pédagogique en prenant appui sur les erreurs récurrentes observées chez les élèves. Cette recherche s'inscrit dans un processus d'échanges entre un laboratoire de psychologie cognitive et des 
professionnels du monde de l'éducation. II s'agit d'une véritable immersion dans l'univers scolaire. Elle propose une démarche pédagogique innovante d'apprentissage à l'inhibition en mathématiques et en orthographe avec des élèves de 6 à 11 ans.

Dans de nombreux modèles de la psychologie du développement, l'inhibition, clef de voûte du développement cognitif, est fondamentale (Dempster, 1992; Dempster et Corkill, 1999; Houdé, 2000, 2007, 2011; McDowd, Oseas-Kreger et Filion, 1995). L'inhibition est une forme de contrôle cognitif et comportemental qui permet aux sujets de résister aux habitudes, aux automatismes, aux tentations, aux distractions ou aux interférences, et de s'adapter aux situations complexes par la flexibilité (Houdé, 2000). On comprend alors toute l'importance de ce processus pour un écolier. En effet, pour apprendre à l'école, il ne suffit pas d'accumuler des connaissances, il faut aussi parfois être capable de bloquer temporairement des stratégies surapprises ou automatisées (que l'on a coutume d'appeler des heuristiques ${ }^{1}$ ) qui deviennent inefficaces dans certaines situations. L'inhibition a d'ailleurs un rôle majeur à jouer dans l'acquisition des apprentissages fondamentaux (Dempster et Corkill, 1999; Diamond, Barnett, Thomas et Munro, 2007). Ces capacités d'inhibition se développent progressivement de l'enfance à l'adolescence (Best et Miller, 2010; Chevalier, 2010; Gaux et Boujon, 2007; Huizinga, Dolan et van der Molen, 2006). Ce développement progressif serait lié au cortex préfrontal, dont la maturation s'effectue lentement jusqu'à la fin de l'adolescence (Bunge, Dudukovic, Thomason, Vaidya et Gabrieli, 2002; Romine et Reynolds, 2005).

L'importance du contrôle inhibiteur dans l'acquisition de connaissances (à travers la correction de l'erreur) dans différents domaines, tels que le nombre et le raisonnement, est rapportée dans de nombreuses études (Cassotti et Moutier, 2010; Houdé, 2000; 2004/2011; Houdé et Moutier, 1996; Houdé et al., 2000, 2011; Moutier, 2000; Moutier, Angeard et Houdé, 2002; Moutier et Houdé, 2003). Ceci a alors amené la présente équipe à redéfinir, autrement que ne l'ont fait Jean Piaget et les néo-piagétiens, les stades de la construction de l'intelligence ainsi que les mécanismes de transition d'un stade à l'autre. II en ressort une conception dynamique plutôt que linéaire du développement cognitif (Figure 1). Ainsi, dans ce modèle, le développement est représenté sous forme de vagues qui se chevauchent, illustrant les stratégies en construction dans le cerveau. Une nouvelle stratégie peut entrer

\footnotetext{
${ }^{1}$ Une heuristique est une stratégie cognitivement peu coûteuse qui fonctionne très bien, très souvent, mais pas toujours. Elle est classiquement opposée à l'algorithme, qui est une stratégie qui mène toujours à la bonne réponse, mais qui est souvent cognitivement beaucoup plus coûteuse à mettre en œuvre.
} 
en compétition avec une ancienne, ce qui provoque des erreurs. En effet, la difficulté pour le sujet est d'inhiber dans ce cas la stratégie non pertinente (heuristique) au bénéfice d'une nouvelle stratégie pertinente.
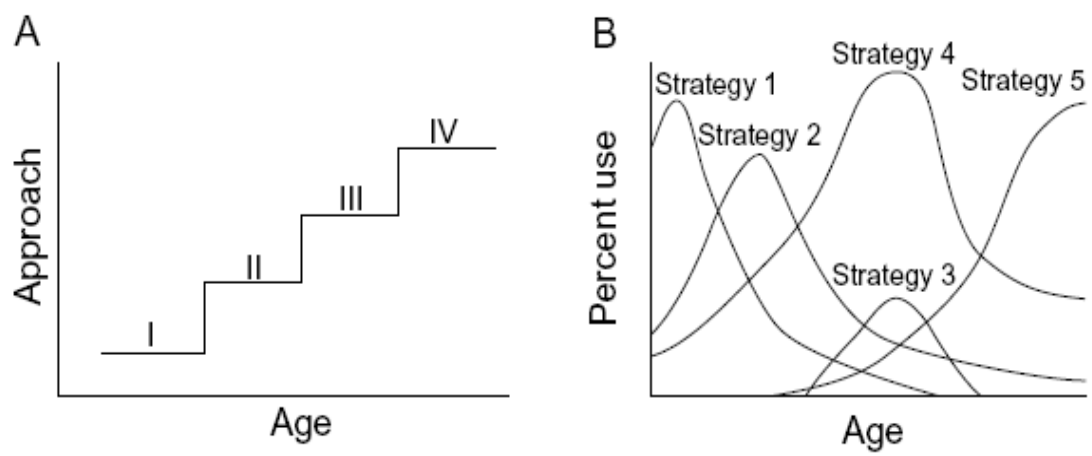

Figure 1. Modèles cognitifs du développement. A. Modèle piagétien en escalier et $B$. Modèle dynamique non-linéaire (adapté de Siegler, 1999).

La tâche classique piagétienne de conservation du nombre a été réinterprétée à la lumière de cette nouvelle théorie (Houdé, 2000; 2004/2011; Houdé et Guichart, 2001; Houdé et al., 2011). Deux rangées composées du même nombre de jetons sont présentées en correspondance terme à terme à l'enfant. L'enfant considère qu'il y en a le même nombre. Par contre, une fois les jetons d'une des rangées écartés, l'enfant, avant 6-7 ans, pense qu'il y a plus de jetons dans la rangée la plus longue (voir Figure 2).

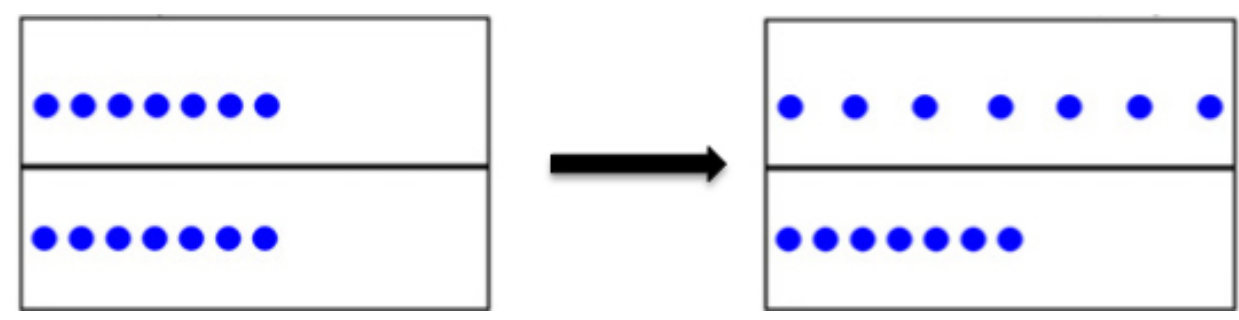

Figure 2. La tâche de conservation du nombre de Piaget. La question posée à l'enfant est: "Y a-t-il le même nombre de jetons dans les rangées du haut et du bas? ». Cette question est posée à deux reprises : dans un premier temps, lorsque les deux rangées ont la même longueur (à gauche), puis dans un second temps, lorsque les jetons d'une des rangées sont progressivement écartés vers la droite devant l'enfant (à droite). La plupart des enfants répondent correctement à la première question, mais avant l'âge de 6-7 ans, ils se trompent généralement à la seconde et déclarent qu'il y a plus de jetons dans la rangée du haut après l'écartement des jetons. 
Lors de la réalisation de cette tâche, l'enfant se trouve face à un conflit perceptivo-cognitif. La difficulté tient ici au fait que deux stratégies entrent en compétition et se télescopent: une heuristique visuospatiale "longueurégale-nombre » et un algorithme de comptage. L'hypothèse des chercheurs est que l'échec de l'enfant serait lié à un défaut d'inhibition de cette heuristique plutôt qu'à un défaut des compétences numériques. Très souvent, dans notre vie de tous les jours, la longueur varie avec le nombre et le cerveau de l'enfant détecte très tôt ce type de régularité visuelle (Houdé, 2004/2011). Dans le matériel pédagogique utilisé en classe par exemple, cette régularité est renforcée, car la suite de chiffres de 1 à 9 est généralement illustrée par des alignements d'objets occupant un espace de plus en plus long. Or, pour réussir la tâche de conservation du nombre, l'enfant doit inhiber l'heuristique "longueur-égale-nombre " qui lui est familière et qui se révèle souvent efficace.

Une étude récente en imagerie par résonance magnétique fonctionnelle (IRMf) chez des enfants âgés entre 5 et 10 ans a montré que la réussite de cette tâche mobilise un large réseau de neurones situés dans le cortex pariéto-frontal bilatéral (Houdé et al., 2011). Ce réseau est connu pour être impliqué à la fois dans les capacités numériques - notamment le sillon intrapariétal - et dans les capacités d'inhibition et de changement de stratégie en mémoire de travail (Figure 3). Cette mobilisation n'apparaît que chez les enfants les plus âgés ou chez des enfants plus jeunes qui commencent à réussir la tâche piagétienne (les enfants dits intermédiaires). C'est donc cette capacité d'inhibition cognitive que le cerveau doit construire pour devenir plus efficace et déjouer les pièges perceptifs de la situation expérimentale mettant en conflit deux stratégies.

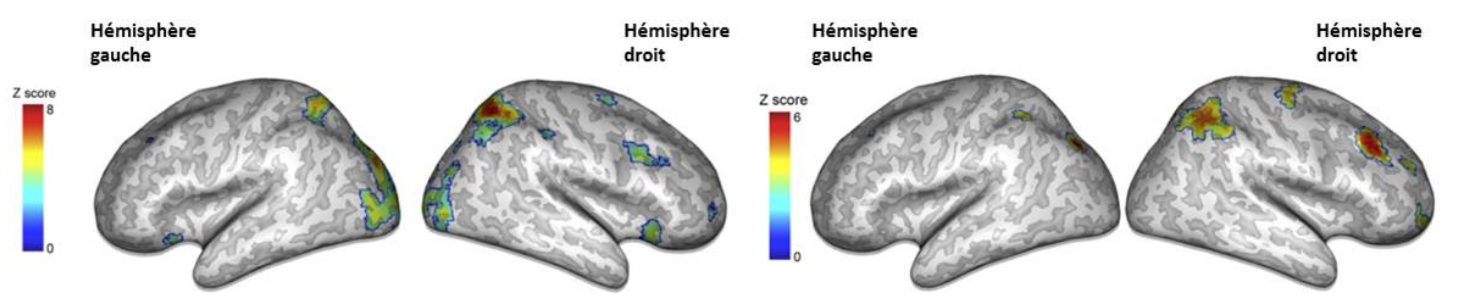

Figure 3. Illustration de la tâche piagétienne en IRMf (Houdé et al., 2011). Dans le cerveau des enfants de 9 à 10 ans qui réussissent systématiquement la tâche présentée à la Figure 2, un réseau de neurones s'active dans le cortex pariétofrontal bilatéral (à gauche). Ce réseau est aussi partiellement activé chez des enfants plus jeunes de moins de 7 ans (enfants intermédiaires) qui commencent à répondre correctement (à droite). 
Dans une perspective neuropédagogique, Houdé et ses collaborateurs ont élaboré une méthodologie expérimentale en laboratoire reposant sur la détection d'un conflit entre une bonne et une mauvaise réponse (entre une heuristique et un algorithme) et l'apprentissage au contrôle inhibiteur. Cette méthode, basée sur l'apprentissage à l'inhibition, a permis de montrer, dans diverses situations, l'utilité d'exercer le sujet (enfant, adolescent ou adulte) à activer la stratégie pertinente (algorithme), mais aussi et surtout à inhiber celle qui ne l'est pas (heuristique) pour résoudre un problème donné. La méthode ici est l'apprentissage expérimental, c'est-à-dire une sorte de " pédagogie de laboratoire " basée sur l'apprentissage à l'inhibition. Le sujet devait apprendre à corriger ses erreurs à l'aide d'alertes exécutives verbales (par exemple: "Attention, dans ce type de tâche, il y a un piège. II faut faire très attention. ») et d'un dispositif didactique expérimental. L'action et la manipulation sont importantes dans les processus pédagogiques: " on apprend aussi avec ses mains " surtout chez les plus jeunes (GoldinMeadow et Wagner, 2005; Lubin, Poirel, Rossi, Pineau et Houdé, 2009; Lubin et al., 2010). Ainsi, afin d'apprendre au sujet à inhiber l'heuristique pour activer l'algorithme, on lui fait manipuler un dispositif didactique (alertes exécutives visuo-spatiales constituées d'un attrape-piège et de cartes réponses colorées : voir Figure 4).

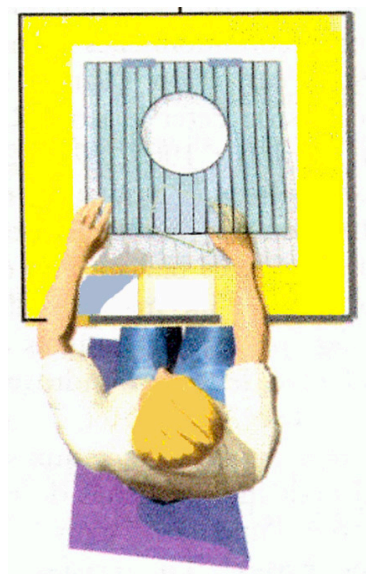

Figure 4. Dispositif didactique expérimental que manipule le sujet pendant l'apprentissage à l'inhibition. L'attrape-piège est une symbolisation du répertoire de schèmes composé d'une partie hachurée transparente à soulever qui matérialise le processus attentionnel d'inhibition et d'une partie ronde centrale découpée qui représente le processus attentionnel d'activation. Le sujet doit soulever la partie hachurée pour y placer la carte réponse rouge liée à l'heuristique : le piège est ainsi « attrapé ». II doit ensuite positionner la carte réponse verte (symbolisation de la stratégie algorithmique) dans la partie centrale. 
L'efficacité de ce type d'apprentissage a été mise en évidence au niveau comportemental dans diverses situations et à des âges différents (Cassotti et Moutier, 2010; Houdé et Moutier, 1996; Moutier, 2000; Moutier et al., 2002; Moutier et Houdé, 2003). Ce paradigme d'apprentissage a aussi été décliné en imagerie cérébrale afin d'observer ce qui se passe dans le cerveau des adultes avant et après l'apprentissage de l'inhibition d'une heuristique, c'està-dire avant et après la correction d'une erreur de raisonnement (Houdé et al., 2000). Les résultats obtenus suite à l'apprentissage à l'inhibition ont montré une reconfiguration - ou plasticité - des réseaux cérébraux, de la partie postérieure du cerveau à sa partie antérieure - dite préfrontale -, impliquée dans le contrôle cognitif.

La capacité d'inhibition, centrale à l'école primaire, demeure en outre importante tout au long du cursus scolaire. Masson, Potvin, Riopel et Brault Foisy (2012) ont d'ailleurs observé que les étudiants en sciences devaient apprendre à inhiber certaines conceptions erronées pour progresser dans l'apprentissage des principes d'électricité. En effet, face à des schémas incorrects de systèmes électriques, les experts mobilisent notamment une zone cérébrale située dans le cortex préfrontal, spécialisée dans la détection des erreurs. Ils ont ainsi repéré le piège. Ce n'est qu'à partir de là qu'ils sont capables de bloquer la réponse intuitive inexacte pour trouver la bonne réponse. Selon ces chercheurs, les enseignants devraient davantage apprendre à leurs élèves à reconnaître les pièges.

Les résultats des études exposées ici incitent donc, face aux blocages, erreurs ou difficultés de certains élèves, à transférer au " monde de la classe " des outils éprouvés en laboratoire avec des techniques comportementales et d'imagerie cérébrale. On peut dès lors imaginer qu'audelà des apprentissages logico-mathématiques en tant que tels, la pédagogie à l'école puisse plus systématiquement porter sur cette capacité à changer rapidement de stratégie de raisonnement et à mobiliser des réseaux neuronaux différents pour résoudre une tâche. Ne peut-on pas faire bénéficier les élèves d'une pédagogie de "l'inhibition » et du contrôle cognitif?

Pour cela, nous avons débuté, depuis 2009, un partenariat avec les professionnels du terrain au sein d'un Groupe Formation Action (GFA) intitulé " pédagogie du contrôle cognitif " créé par l'Inspection Académique du Calvados (France). Ce GFA est composé d'une douzaine d'enseignants volontaires, de conseillers pédagogiques, d'inspecteurs de circonscription et de chercheurs de notre équipe (LaPsyDÉ). L'objectif de cette initiative neuroéducative était de mettre en place des démarches psychopédagogiques innovantes en " co-concevant " des séquences d'apprentissage à l'inhibition 
visant à bloquer des stratégies erronées, mais courantes (heuristiques) dans la vie scolaire de la petite section de maternelle au CM2 (Rossi et al., 2010, 2011, 2012a, 2012b). Durant ces trois années, plusieurs stages ont été organisés à l'Inspection d'Académie du Calvados. Lors de ces rencontres, les enseignants ont été formés à l'importance du contrôle inhibiteur dans les apprentissages et à notre méthodologie de laboratoire afin de la mettre en application avec leurs élèves. Ensuite, ils ont été invités à repérer, au sein de leur propre classe, des situations scolaires de conflits cognitifs étant sources d'erreurs fréquentes et dans lesquelles entrent en compétition une heuristique et un algorithme. Après avoir identifié ces situations-problèmes, nous avons décidé d'y remédier en proposant de tester l'efficacité d'une méthode inspirée de l'apprentissage à l'inhibition développé par l'équipe de Houdé. Nous avons alors créé deux types de séquences pédagogiques portant sur un apprentissage classique et un apprentissage à l'inhibition. Dans la situation classique, l'enseignant focalise l'attention de l'élève sur " ce qu'il faut faire », sur la stratégie adéquate (algorithme). Dans la situation d'apprentissage à l'inhibition, l'enseignant indique la stratégie correcte, mais alerte également explicitement l'élève sur " ce qu'il ne faut pas faire », sur le piège (heuristique) à éviter (en utilisant des alertes verbales et un matériel didactique expérimental à manipuler).

Nous présenterons dans cet article deux situations d'apprentissage développées dans ce GFA, dans lesquelles entrent en compétition une heuristique et un algorithme : l'une en mathématiques et l'autre en orthographe. Les objectifs de cette recherche sont les suivants : 1- éprouver sur le terrain une méthodologie innovante de laboratoire afin d'observer si elle peut s'appliquer dans les classes et 2- mettre en évidence dans des situations scolaires variées, auprès d'élèves d'âges différents, qu'un apprentissage à l'inhibition permettant explicitement aux élèves de repérer les pièges et de les éviter devrait être plus efficace qu'un apprentissage classique. Nous avons ainsi mis en place un protocole expérimental d'apprentissage qui se déroule en trois temps : d'abord, un pré-test portant sur une tâche présentant une situation-problème en mathématiques ou en orthographe, puis une phase d'apprentissage (inhibition ou classique) basée sur le même type de situation-problème, mais présentée dans un autre contexte et enfin, un post-test (identique au pré-test) pour évaluer l'efficacité de l'apprentissage. Nous faisons donc l'hypothèse qu'un apprentissage à l'inhibition devrait être plus bénéfique qu'un apprentissage classique, à la fois dans une séquence de mathématiques mais aussi d'orthographe. 


\section{2. Étude 1 : l'apprentissage de la dizaine chez des élèves de 6-7 ans}

Dès la fin de la maternelle, l'élève acquiert la numération chiffrée. Lors de la seconde année, en cours préparatoire (CP), il commence à apprendre la base 10 - le système décimal - et découvre l'écriture des nombres à deux chiffres et les notions de dizaine et d'unité. Acquérir une compréhension de la base 10 des nombres est nécessaire pour pouvoir réaliser des calculs sur des nombres supérieurs à dix. Néanmoins, cet apprentissage est souvent long et laborieux (Fuson, Smith et Cicero, 1997).

Lors de la découverte des nombres à deux chiffres, l'élève généralise les connaissances qu'il a initialement des nombres à un chiffre à celui des nombres à deux chiffres. Cette généralisation peut, dans ce cas, le mener à l'erreur. En effet, alors qu'il semble comprendre l'équivalence entre 10 unités et une dizaine, il se trompe très souvent lorsqu'on lui demande de comparer deux collections présentées dans des unités différentes. Par exemple, lorsqu'on lui présente 18 unités par rapport à 2 dizaines, il dira que la collection de 18 unités est plus grande que celle contenant deux dizaines. Cette réponse « $18>2$ » est effectivement correcte quand on est face à des quantités présentées dans la même unité, mais dans ce cas précis où les quantités sont dans des unités différentes, elle devient incorrecte.

Ce type d'erreurs robustes nous amène à repenser l'apprentissage dans le cadre du modèle post-piagétien proposé par Houdé (2000) en termes de stratégies concurrentes : une stratégie adéquate (algorithme : « je transforme si nécessaire dans la même unité avant de comparer ») et une stratégie erronée (heuristique : " je compare directement les nombres sans vérifier qu'ils sont dans la même unité "). La difficulté systématique des élèves ici semble être d'inhiber l'heuristique de comparaison brute des nombres (réponse $18>2$ ) afin d'activer l'algorithme de transformation des éléments du problème dans la même unité $(18<20)$, algorithme qu'ils ont pourtant déjà appris. Cette erreur pourrait ne pas être liée à un défaut de compréhension de la notion, mais plutôt à un défaut d'inhibition d'une connaissance antérieure, qui devient dans ce cas précis une heuristique qui ne fonctionne pas.

Aussi, nous faisons l'hypothèse qu'un apprentissage " classique ", centré exclusivement sur la procédure algorithmique (rappel de la règle), sera moins efficace qu'un apprentissage " à l'inhibition », focalisé sur le piège et faisant appel à des alertes exécutives verbales ("Attention au piège ! ") ainsi qu'à la manipulation d'un dispositif didactique avec lequel l'élève agit individuellement. Pour cela, nous avons mis en place auprès d'élèves de 6-7 ans ces deux types d'apprentissage pour pouvoir évaluer l'efficacité de notre méthode. 


\subsection{Méthode}

\subsubsection{Participants}

Cette étude porte sur 45 élèves (âge moyen : $7.4 \pm 0.6$ ans, 22 garçons) de deux classes de Cours Préparatoire (CP) de deux groupes scolaires de Caen et sa périphérie (Calvados, France). Les parents et les enfants ont donné leur consentement pour participer à cette étude.

\subsubsection{Procédure générale}

Les interventions se sont déroulées lors d'une semaine en fin d'année scolaire : le prétest a eu lieu le jour 1 (un vendredi), les séquences d'apprentissage ont été réalisées les jours 2, 3 et 4 (les lundi, mardi et jeudi) et le post-test, le jour 5 (le vendredi). Après le pré-test, les élèves ont été répartis au hasard dans deux groupes d'apprentissage : l'apprentissage à l'inhibition ou l'apprentissage classique.

\section{Pré-test}

Pour le pré-test, un exercice individuel sur papier a été distribué à la classe entière. Cet exercice était constitué de 15 items. L'élève devait entourer la case contenant le plus d'éléments. Parmi ces 15 items, 5 items pièges (ex : 4 dizaines vs. 35 unités), 5 items sans piège (ex: 3 dizaines vs. 35 unités) et 5 items distracteurs (ex: 4 dizaines vs. 3 dizaines) étaient proposés dans un ordre aléatoire. L'ordre de présentation des items a été contrebalancé.

Nous avons considéré comme éligibles à l'apprentissage les élèves qui échouent aux items pièges alors qu'ils réussissent les autres items. Leur score au pré-test était donc inférieur à $50 \%$ de bonnes réponses pour les items pièges (score $\leq$ à 2 sur 5 ) et supérieur à $50 \%$ de bonnes réponses pour les items sans piège et les items distracteurs (scores $\geq$ à 4 sur 5 ). Cependant, pour des raisons d'organisation de classe, tous les élèves (même ceux ne répondant pas à ce critère) ont été soumis aux apprentissages pour respecter l'équilibre interne de la classe et ne pas faire de discrimination entre les élèves éligibles ou non au protocole.

\section{Apprentissages}

Après le pré-test, les différentes séances d'apprentissage ont été effectuées en demi-classe (entre 10 et 12 élèves). Pendant ces séances, l'autre moitié de la classe était prise en charge par un enseignant remplaçant et effectuait une autre activité. Nous avons réparti équitablement les élèves éligibles au 
protocole dans les 2 types d'apprentissage : apprentissage à l'inhibition et apprentissage classique. Chaque apprentissage était constitué de 3 séances pédagogiques d'une durée maximum de 30 minutes.

\section{Apprentissage à l'inhibition}

Durant la première séance pédagogique, l'enseignant propose une situation problème :

"Paul et Kévin vont dans la forêt ramasser des brindilles pour allumer le feu. Paul met toutes ses brindilles dans son sac sans le ranger, mais Kévin décide de faire des fagots de 10 brindilles pour les utiliser plus facilement. Au retour de leur promenade. Paul a 30 brindilles et Kévin a 4 fagots. Qui a le plus de brindilles ? »

Après s'être assuré qu'ils n'avaient pas de problèmes de compréhension, les élèves sont invités à répondre par écrit et à illustrer comment ils ont procédé sur une feuille blanche. Une mise en commun est ensuite réalisée. Les productions des élèves sont alors affichées au tableau et regroupées par stratégie. Un élève de chaque groupe explique la stratégie utilisée. L'enseignant rappelle les procédures proposées par les groupes et complète, le cas échéant, pour mettre en évidence la stratégie correcte. Pour conclure la première séance, l'enseignant met en évidence le piège dans cette situation :

« Attention, dans ce type d'exercice, il y a un piège ! Le piège c'est de comparer seulement les nombres, sans avoir vérifié avant que l'on parle bien de la même chose! »

Lors de la seconde séance, l'enseignant rappelle la situation problème proposée la veille, la stratégie correcte pour la résolution du problème ainsi que le piège à éviter. Le matériel est alors présenté (Figure 5) par l'enseignant. II se compose d'un attrape-piège (partie hachurée sous laquelle le piège peut être placé et un rond central dans lequel la réponse correcte peut être disposée) et de cartes réponses (une carte rouge en forme d'arrêt symbolisant l'heuristique/piège et une carte verte symbolisant l'algorithme).

"Pour ne pas tomber dans le piège, vous allez utiliser un attrapepiège et des cartes stratégies. Vous aurez une carte verte " à deux faces " qui correspond à ce qu'il faut faire et une carte rouge, symbolisant le piège, qui signifie STOP, arrêtez-vous! Quand on compare sans vérifier, on peut tomber dans un piège! C'est ce qu'il ne faut pas faire. Je ne dois pas aller trop vite! Si je vais trop vite, je peux tomber dans le piège! Le piège, c'est de comparer les nombres sans avoir vérifié avant qu'on est dans les mêmes unités, qu'on compte bien la même chose! Le piège, c'est de se dire " comme d'habitude, le grand nombre, c'est là où il y en 
a le plus ». Mais, attention, je m'arrête et je réfléchis, il peut y avoir un piège, je n'ai pas vérifié (carte alerte "Stop »: Je compare sans vérifier). Je vais vous donner aussi une carte-réponse verte à deux faces qui correspond à ce qu'il faut faire : Sur une face, vous avez "si c'est pareil, je compare " et sur l'autre face, " si ce n'est pas pareil je transforme ». [...] Pour ne pas tomber dans le piège, on va mettre la carte rouge " je compare sans vérifier " qui correspond au piège : sous les rayures, c'est l'attrape-piège. C'est ce qu'il ne faut pas faire! [...] Les bonnes stratégies, c'est celles qui correspondent aux faces de la carte verte. La carte verte, on la placera au milieu de l'attrape-piège, " du bon côté », suivant les situations, parce que c'est ce qu'il faut faire. [...]».

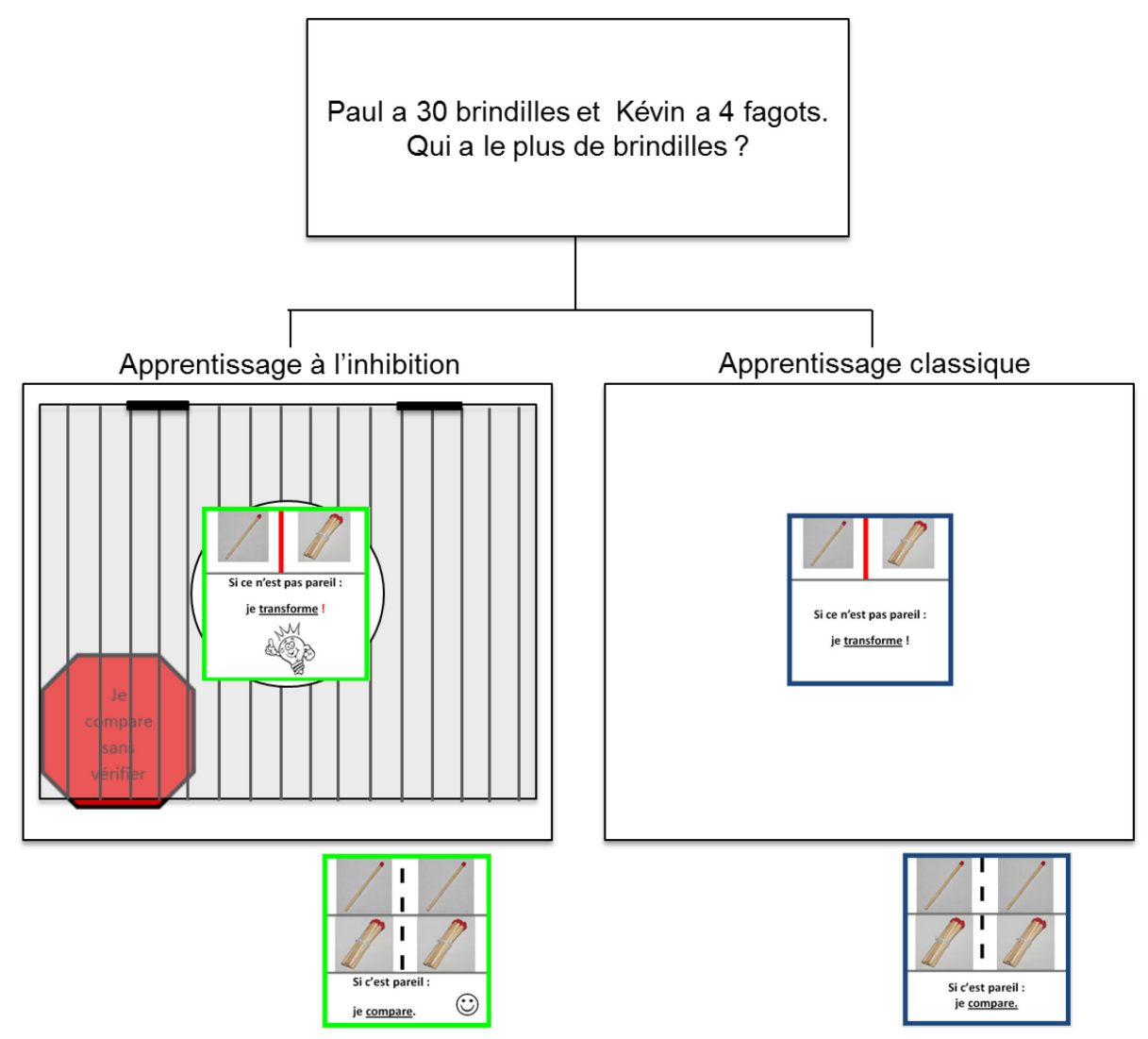

Figure 5. Dispositif didactique expérimental utilisé lors des séances d'apprentissage. À gauche : le dispositif de l'apprentissage à l'inhibition avec l'attrape-piège et les cartes réponses. La carte piège en forme de panneau STOP est rouge et la carte-réponse correcte est verte avec au recto " si je compte dans la même unité, je compare " et au verso "si je compte dans des unités différentes, je transforme puis je compare ". À droite : le dispositif de l'apprentissage classique avec la carte réponse correcte en bleu. 
Chaque élève dispose d'un matériel individuel pour s'exercer. Les élèves manipulent le matériel et placent les différentes cartes dans l'attrape-piège pour illustrer la situation initiale. Ensuite, d'autres situations similaires sont proposées. Les élèves doivent répondre à la question en s'aidant du matériel. Chaque situation est alors reprise par l'enseignant.

Lors de la troisième et dernière séance, un travail individuel est proposé sur une fiche. Sur la fiche, six situations sont illustrées (ex. : Jules a 5 fagots. Pauline a 46 brindilles). Les élèves doivent entourer le personnage qui en a le plus. Ils sont incités à utiliser la carte piège, la carte stratégie et l'attrapepiège s'ils le souhaitent. Lors de la correction collective, chaque situation est reprise en explicitant l'unité dans laquelle on compare et en utilisant le matériel.

\section{Apprentissage classique}

L'apprentissage classique se déroule strictement de la même manière sauf que 1-l'enseignant n'évoque jamais le «piège » et concentre ses interventions pédagogiques sur la bonne façon de faire pour résoudre le problème " transformer avant toute comparaison dans la même unité ", 2- le matériel contient uniquement la carte-réponse correcte bleue (Figure 5).

\section{Post-test}

Le même exercice utilisé en prétest était proposé en post-test à la classe entière un jour après la dernière séance d'apprentissage.

\subsection{Résultats}

Nous avons traité uniquement les résultats des élèves éligibles au protocole, à savoir ceux qui échouaient aux items pièges et réussissaient aux items sans piège et distracteurs lors du pré-test. 24 élèves (âge moyen : $7.3 \pm 0.7$ ans, 11 garçons), soit $53 \%$ des élèves interrogés, ont été retenus. Ainsi, plus de la moitié des élèves se trompent, car ils ne parviennent pas à inhiber l'heuristique. Parmi les autres élèves, certains ne tombaient pas dans le piège (score supérieur à $50 \%$ de bonnes réponses aux items pièges) et d'autres échouaient à tous les items (score inférieur à $50 \%$ de bonnes réponses dans tous les types d'items).

Les 24 élèves ont été soumis aux deux tests (pré-test et post-test). La moitié des sujets a été exposée à l'apprentissage classique et l'autre moitié à l'apprentissage à l'inhibition. Dans les deux types d'apprentissages, chaque 
élève peut obtenir une note maximale de 5 points pour chaque type d'items. Notre variable dépendante est le score de réussite en pourcentage à chaque test. Afin de tester l'effet de ces apprentissages, nous avons réalisé des analyses de la Variance (ANOVA) séparées (les conditions d'application sont respectées) comportant une variable inter: Apprentissage (inhibition vs. classique) et une variable intra: Test (pré-test vs. post-test) sur les items pièges et les items sans-piège. Ces analyses ont été complétées par des comparaisons planifiées a priori lorsque l'effet d'interaction n'était pas significatif. Pour chacune de ces analyses, nous avons reporté la taille de l'effet (éta-carré partiel pour l'ANOVA ou $d$ de Cohen pour les différences de moyennes).

\subsubsection{Items pièges}

On observe que tous les élèves bénéficient de l'apprentissage, car les performances sont meilleures au post-test $(\mathrm{m}=70.8 \%)$ qu'au pré-test $(m=12.5 \%), F(1,22)=71.10, p<.00001, \eta_{p}{ }^{2}=.76$. Cependant, nous n'observons pas d'effet du type d'apprentissage, $\mathrm{F}(1,22)=3.66, p=.06$, et d'interaction entre ces deux facteurs, $F(1,22)=3.72, p=.06$. Les comparaisons planifiées révèlent qu'il n'y a pas de différence significative entre les deux groupes au pré-test (mclassique $=10 \%$ et minhibition $=15 \%$ ), $t(22)$ $<1$. Par contre, il y a une différence significative entre les deux groupes au post-test, $\mathrm{t}(22)=2.10, \mathrm{p}<.05, d=.86$. Les élèves du groupe inhibition ont de meilleures performances $(m=87 \%)$ que ceux du groupe classique $(m=55 \%)$ après l'apprentissage (Figure 6).

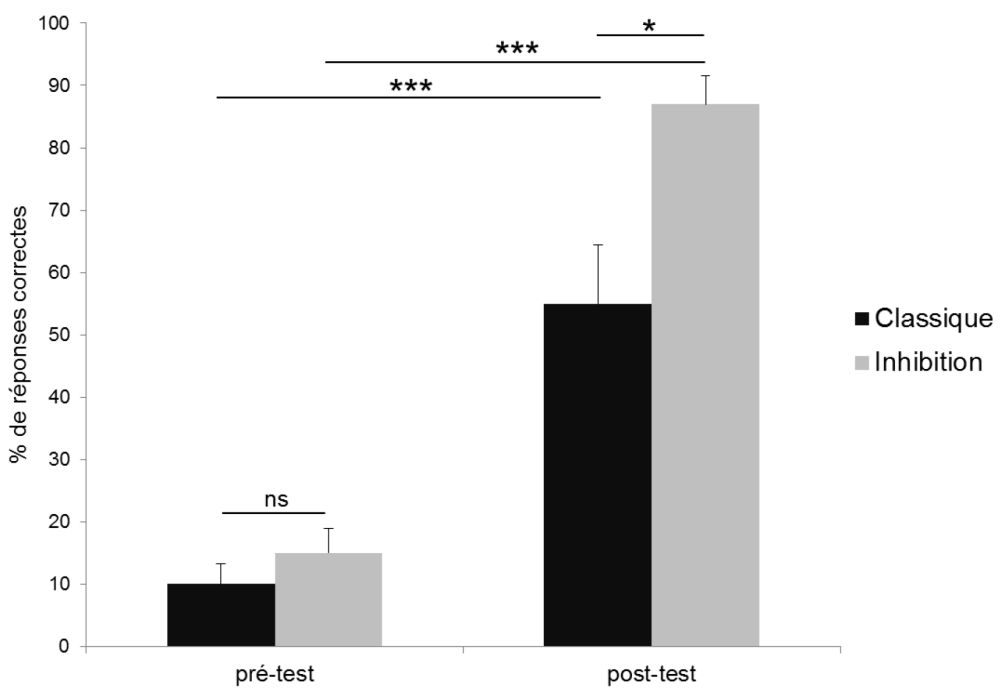

Figure 6. Pourcentage de réponses correctes lors des pré- et post-tests en fonction de l'apprentissage ( $n s=$ non-significatif ; ${ }^{*} p<.05$; ${ }^{* *} p<.001$ ). 


\subsubsection{Items sans-piège}

Les résultats ne montrent pas d'effet significatif du test, $F(1,22)=3.07$ $p=.10$, ni d'effet de l'apprentissage, $F(1,22)=2.42 p=.14$, ou d'interaction entre ces deux facteurs, $F(1,22)=1.92 p=.18$. Les élèves ont de bonnes performances aux items sans piège, quel que soit le test ou le type d'apprentissage (moyennes supérieures à $75 \%$ de bonnes réponses).

\subsection{Discussion}

Les résultats de cette première étude psychopédagogique dans le domaine des mathématiques mettent en évidence l'avantage d'un apprentissage à l'inhibition par rapport à un apprentissage classique. En effet, les élèves du groupe inhibition ont amélioré leur performance de façon plus importante que ceux du groupe classique. Ainsi, orienter l'attention de l'élève sur le piège à éviter et lui donner des outils pédagogiques matérialisant le piège et son blocage parait être un atout pour celui-ci. Les élèves auraient des difficultés à inhiber la connaissance antérieure qu'ils ont de la chaine numérique (le nombre le plus grand est celui qui est le plus important dans la chaine numérique) pour activer la nouvelle règle qu'ils viennent d'apprendre concernant l'équivalence " 10 unités = une dizaine ». L'apprentissage à l'inhibition les aiderait à dépasser ce conflit.

Bien entendu, cela ne concerne pas tous les élèves, car nous avons observé que la moitié d'entre eux environ ne tombent pas dans ce piège. Cependant, il y a souvent à un moment donné de l'apprentissage, une "fenêtre temporelle " durant laquelle certains élèves n'y arrivent pas et souvent l'enseignant s'interroge, car ils paraissent pourtant comprendre la notion. Ainsi, un apprentissage à l'inhibition peut dans ce cas être profitable, car il permet aux élèves de surmonter le conflit cognitif et de comprendre plus rapidement leurs erreurs en déjouant le piège.

Cette méthodologie pourrait ensuite être réinvestie avec des élèves de 8 à 11 ans lors des situations similaires, comme l'apprentissage des unités de mesure (millimètres, mètres, etc.), ou bien encore dans d'autres matières scolaires comme l'orthographe. Nous présenterons dans le cadre de l'étude 2, une situation de ce type qui pose problème aux élèves de 1011 ans. 


\section{3. Étude 2 : l'acquisition de l'orthographe grammaticale chez des élèves de 10-11 ans}

L'acquisition de l'orthographe est un domaine particulièrement étudié depuis une quinzaine d'années. La majorité des recherches portent sur une souscomposante de l'orthographe grammaticale qui fait l'objet de cette étude : l'accord des noms et des verbes, et plus précisément l'accord en nombre en français. Cet apprentissage est source de difficultés pour de nombreux enfants de 10 à 11 ans. Ces difficultés proviennent notamment du fait que les marques grammaticales sont inaudibles dans la langue française (ex.: " l'enfant joue au ballon / les enfants jouent au ballon ") en plus d'être différentes selon la classe syntaxique (c.-à-d. pluriel des noms versus pluriel des verbes).

Diverses études (Fayol, Hupet et Largy, 1999; Largy, 2001; Totereau, Barrouillet et Fayol, 1998) dévoilent l'existence de différentes étapes de l'acquisition de l'orthographe grammaticale. Au cours de la première étape, les enfants ne produisent pas de marque d'accord (ou écrivent de façon phonétique), mais ont néanmoins des connaissances passives sur l'accord leur permettant d'associer un mot écrit et une image ou de détecter une erreur d'accord. Lors de la deuxième étape, les enfants mettent en place la procédure de production de l'accord, ce qui nécessite une quantité importante de ressources cognitives et qui aboutit dans certains cas à des erreurs de confusion de marques (c.-à-d. les erreurs de sur-généralisation : " ils manges »). Enfin, c'est lors d'une troisième étape que la procédure de production de l'accord est automatisée et ne nécessite plus qu'une quantité réduite de ressources. L'hypothèse défendue par différents auteurs est celle de l'application d'une procédure algorithmique de type " quand le nom est au pluriel, je dois mettre un " $s$ ", " quand le sujet est au pluriel, je dois mettre le verbe au pluriel » étayée par les pratiques pédagogiques en classe (Fayol et al., 1999; Largy, 2001). Cette procédure devient, très vite, rapide et automatisée.

Cependant, une fois la règle acquise, maîtrisée puis automatisée, des erreurs persistent (p. ex. " je les manges "). Elles nous amènent alors à repenser l'apprentissage dans le cadre du modèle post-piagétien proposé par Houdé (2000) en termes de conflit cognitif entre des stratégies concurrentes : la stratégie adéquate (algorithme : " je vérifie et j'accorde avec le mot qui commande le verbe ") et la stratégie erronée (heuristique : "comme je ne vérifie pas, j'accorde avec le mot placé devant ») que l'enfant doit inhiber.

Nous faisons l'hypothèse qu'un apprentissage "classique " centré exclusivement sur la procédure algorithmique (rappel de la règle, utilisation 
du codage habituel de la classe : on encadre le verbe et on souligne le sujet) sera moins efficace qu'un apprentissage à l'inhibition focalisé sur le piège et faisant appel à des alertes exécutives verbales ("Attention au piège! ") et à la manipulation d'un dispositif didactique individuel, constitué d'un attrapepiège et de cartes réponses.

\subsection{Méthode}

\subsubsection{Participants}

L'étude porte sur 53 élèves (âge moyen: $10.8 \pm 0.3$ ans, 22 garçons) de deux classes de Cours Moyen deuxième année (CM2) de deux groupes scolaires de Caen et sa périphérie (Calvados, France). Les parents et les enfants ont donné leur consentement pour participer à cette étude.

\subsubsection{Procédure générale}

Le protocole d'apprentissage est proposé dans deux classes, l'une réalisant l'apprentissage à l'inhibition, l'autre l'apprentissage classique. Il est composé de deux séances réalisées durant deux jours en fin d'année scolaire. Le prétest et la séance 1 d'apprentissage le premier jour, puis la séance 2 ainsi que le post-test le second. Chaque séance d'apprentissage dure environ 20 minutes.

\section{Pré-test}

II s'agit d'une dictée contenant 5 items pièges ("le maître les regarde ") et 5 items contrôles ( "les enfants ").

\section{Apprentissages}

\section{Apprentissage à l'inhibition}

Lors de la première séance, l'enseignant propose aux élèves de compléter une fiche d'exercices à trous composée de 5 items ( 2 pièges et 3 contrôles). Sans ramasser les productions des élèves, il les interroge sur les diverses façons d'orthographier l'item suivant (" Léo les aime/Léo les aimes/Léo les aiment »). L'enseignant fait ensuite une mise en commun et rappelle la règle à utiliser et identifie le piège.

«Pour accorder un verbe au présent de l'indicatif, je recherche le sujet. Mais attention, le piège c'est d'accorder le verbe au mot qui est juste devant sans avoir vérifié qui est le mot qui commande 
l'accord. Si on ne regarde que le mot juste avant, on peut se tromper et tomber dans un piège $»$.

II présente alors l'attrape-piège et les cartes alertes réponses verte et rouge. « Pour penser à ne pas tomber dans le piège, vous allez utiliser un attrape-piège et des cartes-réponses verte et rouge. Voici la carte verte : c'est la bonne stratégie, c'est ce qu'il faut faire. Elle a deux faces. D'un côté, c'est la règle écrite : « je vérifie et j'accorde avec le mot qui commande le verbe ». C'est la stratégie que je dois adopter : je suis la flèche. Regardez maintenant, de l'autre côté, il y a le pictogramme: j'identifie le verbe et je recherche le mot qui commande le verbe. Voici la carte alerte rouge : c'est la mauvaise stratégie, c'est ce qu'il ne faut pas faire. Elle a deux faces. D'un côté, c'est la règle écrite: "comme je ne vérifie pas, j'accorde avec le mot devant ». C'est la stratégie que je dois rejeter : c'est le sens interdit. Regardez, de l'autre côté, il y a le pictogramme: j'identifie le verbe et j'accorde toujours avec le mot devant. On va utiliser l'attrape-piège. Pour ne pas tomber dans le piège, il faut mettre la carte rouge qui est la mauvaise stratégie sous les rayures et la carte verte qui est la bonne stratégie au milieu dans le rond blanc. Attention! Vous ne devez pas aller trop vite! Si vous allez trop vite, vous risquez de tomber dans le piège! Le piège, c'est de dire : « comme souvent, le verbe s'accorde avec le mot placé juste devant ». Mais non, STOP, il peut y avoir un piège, donc j'utilise mon attrape-piège $»$.

Les élèves corrigent les quatre autres items sur leur feuille en manipulant leur attrape-piège (Figure 7). L'enseignant les écrit au tableau en rappelant la règle utilisée. 


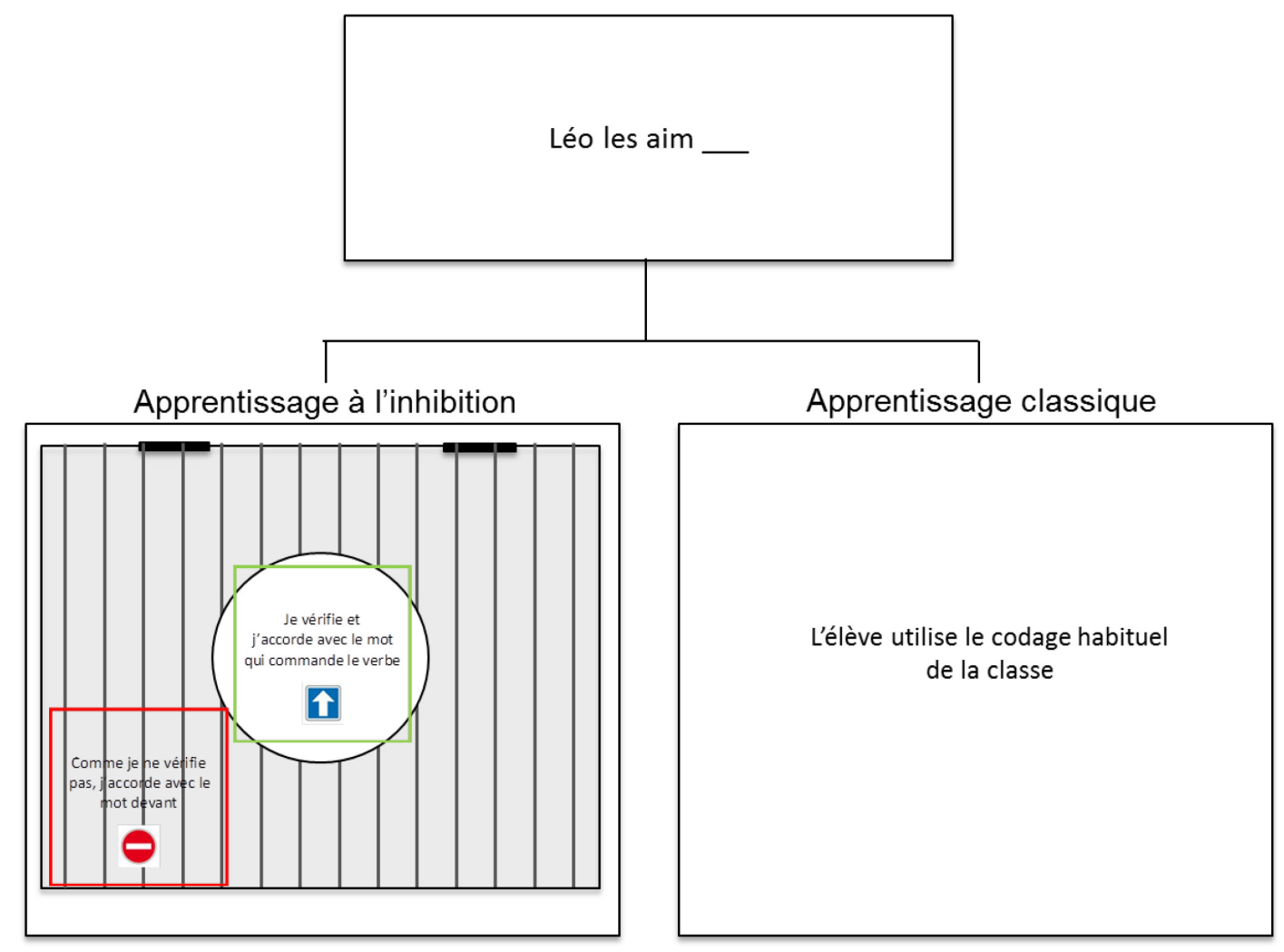

Figure 7. Dispositif didactique expérimental utilisé lors des séances d'apprentissage. À gauche: le dispositif de l'apprentissage à l'inhibition avec l'attrape-piège et les cartes réponses en rouge (la stratégie heuristique) et en vert (la stratégie algorithmique). À droite : le dispositif de l'apprentissage classique.

Lors de la deuxième séance, l'enseignant dicte 5 items (2 pièges et 3 items contrôles). II demande ensuite aux élèves de vérifier si les accords sont corrects en utilisant l'attrape-piège en situation individuelle. II corrige en réalisant une mise en commun phrase par phrase. Puis, il demande aux élèves de compléter une fiche d'exercices à trous comportant 5 items (2 pièges, 3 contrôles) en manipulant l'attrape-piège. Les élèves travaillent ensuite par 2 pour se corriger mutuellement à l'aide de l'attrape-piège en vérifiant les accords sujet/verbe et déterminant/nom. L'enseignant écrit la correction au tableau.

\section{Apprentissage classique}

L'apprentissage classique se déroule strictement de la même manière sauf que 1- l'enseignant n'évoque jamais le " piège », 2- il propose à ses élèves le 
codage habituel de la classe (on encadre le verbe et on souligne le sujet) et 3- il n'utilise pas de matériel (Figure 7).

\section{Post-test}

Le même exercice utilisé en pré-test était proposé en post-test quelques heures après la dernière séance d'apprentissage.

\subsection{Résultats}

Nous avons traité uniquement les résultats des enfants éligibles au protocole, à savoir les enfants qui échouaient aux items pièges (score inférieur à $50 \%$ de bonnes réponses, à savoir $\leq$ à 2 sur 5 ) et réussissaient aux items sans piège (score supérieur à $50 \%$ de bonnes réponses, à savoir $\geq$ à 4 sur 5 ) lors du pré-test. 28 élèves (âge moyen : $10.8 \pm 0.3$ ans, 18 garçons), soit $53 \%$ des élèves interrogés, ont été retenus: 11 bénéficiant de l'apprentissage classique et 17 de l'apprentissage à l'inhibition. Ainsi, plus de la moitié des élèves se trompent, car ils ne parviennent pas à inhiber l'heuristique.

Dans les deux apprentissages, chaque enfant peut acquérir une note maximale de 5 points pour chaque type d'items. Notre variable dépendante est le score de réussite en pourcentage à chaque test. Afin de tester l'effet de ces apprentissages, nous avons réalisé des analyses de variance (ANOVA) séparées (les conditions d'application sont respectées) comportant une variable inter: Apprentissage (inhibition vs. classique) et une variable intra : Test (pré-test vs. post-test) sur les items pièges et les items sans-piège. Ces analyses ont été complétées par des comparaisons a posteriori (test post-hoc Bonferroni) lorsque l'effet d'interaction était significatif. Pour chacune de ces analyses, nous avons reporté la taille de l'effet (éta-carré partiel pour l'ANOVA et d de Cohen pour les différences de moyennes).

\subsubsection{Items pièges}

Nous observons un effet d'interaction entre les facteurs Test et Type d'apprentissage, $F(1,26)=7.70, p=.01, \eta_{p}^{2}=.22$. En effet, les tests post-hoc révèlent qu'il n'y a pas de différence significative entre les deux apprentissages au pré-test ( $m_{\text {classique }}=18.2 \%$ et $\mathrm{m}_{\text {inhibition }}=14 \%, p=.95$ ) alors qu'il $y$ en a une au post-test (mclassique $=49 \%$ et $m_{\text {inhibition }}=75.2 \%, p=.01$, $\mathrm{d}=.98$ ). Les élèves du groupe inhibition ont de meilleures performances que ceux du groupe classique après l'apprentissage (Figure 8). 


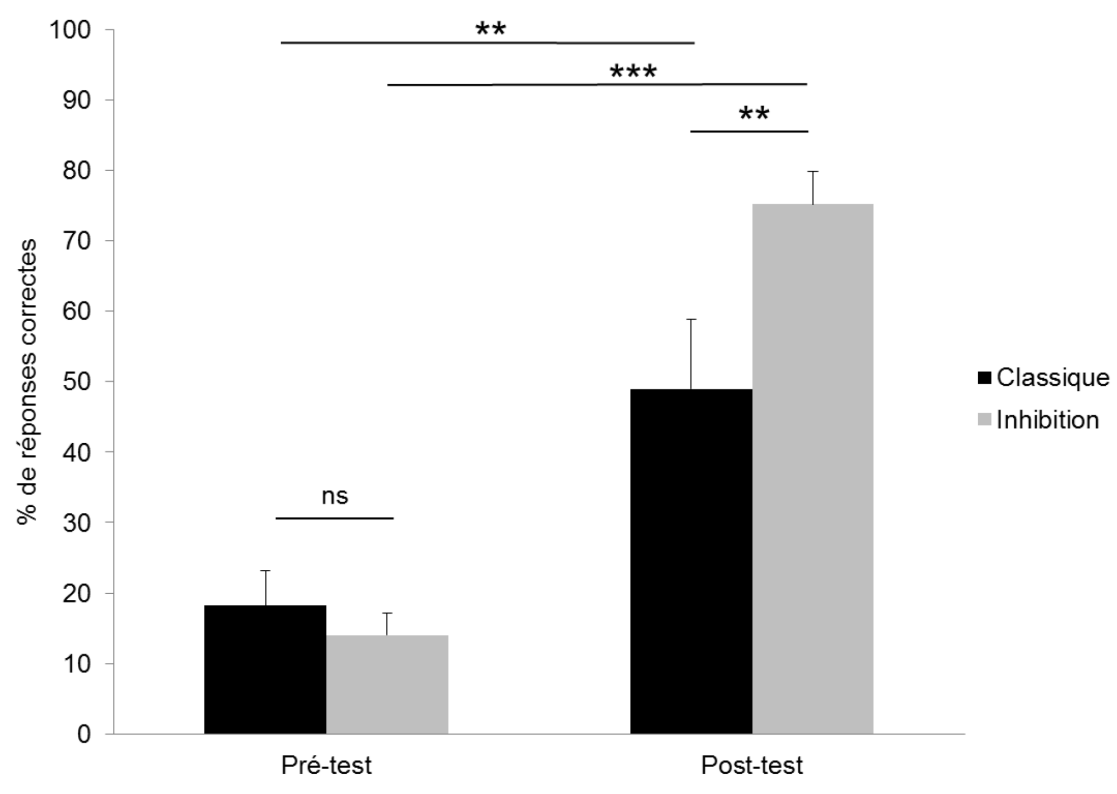

Figure 8. Pourcentage de réponses correctes lors des pré- et post-tests en fonction de l'apprentissage (ns= non-significatif ; ${ }^{* *} p<.01 ;{ }^{* *} p<.001$ ).

\subsubsection{Items sans-piège}

Les résultats ne montrent pas d'effet significatif du test, $F(1,26)=1.22, p=.28$, ni d'effet de l'apprentissage, $F(1,26)=.18, p=.67$, ou d'interaction entre ces deux facteurs, $F(1,26)=.02, p=.89$. Les élèves ont de bonnes performances aux items sans piège, quel que soit le test ou le type d'apprentissage (moyennes supérieures à $75 \%$ de bonnes réponses).

\subsection{Discussion}

Les résultats de cette étude éclairent sous un nouvel angle psychopédagogique le rôle du contrôle cognitif dans l'acquisition de l'orthographe grammaticale chez des élèves de CM2. Ils mettent en évidence l'efficacité de l'apprentissage à l'inhibition qui permet aux élèves de progresser de façon plus importante qu'un apprentissage classique dans des situations qui présentent un piège (ex. : « La cliente les achète »).

Dans ces situations particulières qui présentent un conflit cognitif entre deux savoirs, deux procédures entrent en compétition et l'application de la procédure algorithmique " je vérifie et j'accorde avec le mot qui commande le verbe " (Fayol et al., 1999; Largy, 2001) de l'apprentissage classique n'est pas suffisante, puisque des erreurs persistent chez des élèves qui maîtrisent pourtant cette règle. Ce contexte didactique conflictuel incite donc tous les 
élèves à effectuer un choix de stratégie (heuristique/algorithmique) pour trouver la bonne réponse. Seule la moitié d'entre eux mettront en œuvre la stratégie adéquate. Pour les autres élèves qui bloquent sur le piège, l'enseignant a donc tout intérêt à centrer sa démarche didactique sur un apprentissage à l'inhibition: identifier le piège et mettre en œuvre une réflexion métacognitive avec ses élèves. Repérer et manipuler le piège permet, en effet à l'élève d'exercer sa conscience morphosyntaxique afin d'inhiber la stratégie erronée inférée par la proximité spatiale (" comme je ne vérifie pas, j'accorde avec le mot placé devant ») lui permettant d'éviter de tomber dans le piège. Nos résultats corroborent ainsi les liens qui existent entre la conscience morphosyntaxique et l'apprentissage de l'orthographe grammaticale (Nunes, Bryant et Bindman, 2006; Walker et Hauerwas, 2006).

On pourrait croire que cela ne concerne que les enfants, mais combien d'adultes ne résistent pas à ces pièges en écrivant un message électronique trop rapidement? La pédagogie de l'inhibition est un concept que l'on pourrait décliner auprès de jeunes enfants (Rossi, Lubin, Lanoë et Pineau, 2012b, dans le présent numéro), mais aussi d'adolescents ou d'adultes en formation (Masson et al., 2012).

\section{Conclusion}

Le passage du laboratoire à l'école d'une pédagogie de l'inhibition met en évidence des résultats très prometteurs tout en promulguant une démarche dynamisante qui a suscité l'enthousiasme des enseignants et des chercheurs. En effet, les deux études réalisées dans deux domaines scolaires fondamentaux avec des élèves d'âges différents montrent le bénéfice d'une pédagogie du contrôle cognitif dans les classes ainsi que son caractère transférable. Renforcer une notion scolaire tout en mettant en exergue les stratégies inefficientes, ou "pièges », qu'il faut bloquer à l'aide d'un matériel ludique à manipuler, semble améliorer les performances des élèves de façon plus importante qu'une méthode pédagogique plus classique centrée exclusivement sur l'apprentissage de la stratégie efficiente. Les deux séquences proposées, l'une en mathématiques, l'autre en orthographe, ont ceci de commun qu'elles présentent chacune un conflit cognitif entre deux savoirs: l'un ancien et l'autre nouveau, en construction. C'est le paradoxe créé par le nouveau contexte d'apprentissage qui peut induire chez certains élèves des erreurs.

Nous proposons un dispositif didactique expérimental original et novateur pouvant être utilisé en classe afin d'aider les élèves à surmonter les pièges et les erreurs. La mise en œuvre d'une pédagogie de l'inhibition permet de 
faciliter l'apprentissage des enfants en utilisant un matériel didactique transférable dans différents contextes scolaires. II s'agit dans cet apprentissage non pas d'entraîner les fonctions exécutives per se, mais de faire prendre conscience à l'élève des pièges qu'il peut rencontrer dans sa scolarité tout en lui donnant des outils pour les surmonter. II ne s'agit donc pas d'une méthode portant essentiellement sur l'entraînement des fonctions cognitives (Diamond, 2007, 2011; Gagné et Ainsley, 2002; Gagné et Longpré, 2004; Gagné et Noreau, 2005), mais plutôt d'une approche plus pragmatique de la métacognition qui vise à enseigner aux élèves une méthodologie de travail centrée sur le contrôle cognitif et la détection de conflit dans le cadre d'une situation d'apprentissage ciblée dans un domaine particulier. Apprendre à apprendre est une compétence fondamentale à développer chez tous les élèves afin de favoriser la réussite dans tous les domaines d'apprentissage. L'élève développe ainsi des connaissances réflexives sur ses propres processus mentaux, une réflexion métacognitive (Fernandez-Duque, Baird et Posner, 2000; Shimamura, 2000; Veenman, Van Hout-Wolters et Afflerbach, 2006). De plus, on sait aujourd'hui que la manipulation et l'action sont essentielles en pédagogie (Goldin-Meadow et Wagner, 2005; Lubin et al., 2010). Cette manipulation concrète du matériel didactique par l'élève préfigure les stratégies cognitives à inhiber (et à activer). L'action lui fait prendre conscience des processus mentaux invisibles sur lesquels il peut symboliquement agir. Grâce à cette transposition du jeu à la réalité, il sera mieux outillé pour surmonter les conflits cognitifs rencontrés lors des acquisitions scolaires.

II est vrai que les erreurs robustes évoquées ici ne touchent pas tous les élèves puisqu'un certain nombre d'entre eux surmonteront sans trop de difficulté ces situations conflictuelles. Mais cette pédagogie de l'inhibition s'avère efficace pour les élèves qui persistent dans l'erreur. Elle pourrait ainsi être envisagée dans le cadre d'une remédiation ou d'une prise en charge individualisée auprès des élèves en difficulté d'apprentissage. Mais, on peut également imaginer qu'elle puisse être proposée dès le départ pour aider à la mise en place d'un apprentissage qui présente un piège, un conflit que l'enfant doit surmonter en apprenant à inhiber la stratégie erronée.

II s'agit d'études pionnières réalisées dans un contexte écologique avec les limites expérimentales liées au terrain. Les effectifs peuvent paraître réduits, l'organisation parfois un peu éloignée de ce qui se passe habituellement en classe, mais il nous a fallu nous adapter aux contraintes de la classe, la classe n'étant pas un laboratoire. II serait intéressant d'en évaluer les effets à long terme, ainsi que la reproductibilité à des âges différents et dans d'autres domaines d'apprentissage (par exemple: sciences, géométrie et résolution 
de problème). Par ailleurs, il serait également pertinent d'évaluer le transfert cognitif réalisé par les élèves d'un domaine d'apprentissage à un autre.

Il est essentiel de sensibiliser le terrain éducatif à l'importance du contrôle cognitif dans les apprentissages pour permettre aux élèves d'apprendre à apprendre. Les enfants d'école élémentaire ont un cerveau qui doit encore relever de sérieux défis neurocognitifs: apprendre à s'adapter et parfois changer radicalement de stratégie en résistant aux pièges. L'enseignant est là non seulement pour lui transmettre de nouvelles connaissances, mais aussi et surtout pour l'aider à éviter les pièges et lui faire prendre conscience de ses ressources pour mieux apprendre.

Neurosciences et sciences cognitives apportent aujourd'hui des connaissances nouvelles sur le fonctionnement du cerveau des élèves. Une étape essentielle, mais difficile, demeure l'appropriation des découvertes en neurosciences cognitives par le monde éducatif afin de les réinvestir en classe. Multiplier les recherches et surtout les collaborations entre chercheurs en neuroéducation et professionnels de l'éducation permettra d'aider les élèves, notamment les plus en difficulté, en leur permettant d'accéder aux différents apprentissages scolaires fondamentaux. Observer le cerveau de l'enfant qui apprend afin de le mettre au service du monde scolaire, tel est le nouveau défi de la neuropédagogie du XXIe siècle.

\section{Remerciements}

Nous remercions tout d'abord Messieurs Huchet et Vicet, Inspecteurs d'Académie du Calvados, d'avoir permis la réalisation de ce Groupe Formation Action "pédagogie du contrôle cognitif ». Nous remercions l'ensemble du groupe pour ces trois années riches d'échanges et d'idées: enseignants et conseillers pédagogiques, ainsi que Madame Corinne Sourbet et Monsieur Potdevin, Inspecteurs de l'Education Nationale. Nous remercions tout particulièrement les membres des cycles des apprentissages fondamentaux et des approfondissements pour leur aide dans la mise en place de ce protocole: Patrice Couppey, Corinne Hervé, Joachim Lorusso, Jean-Michel Peres, Laurence Touroult, Emmanuel Vannier et les conseillères pédagogiques Annie Bonnaire, Laurence Bougnon, Françoise Darcieu et Catherine Hardy. Merci également à Patrice Couppey, Corinne Hervé, JeanMichel Peres et Laurence Touroult pour les passations en classe. Merci aussi à tous les élèves de leur participation. Nous tenons également à rendre hommage à $M$. Wisnewski, Inspecteur adjoint d'Académie, sans qui ce groupe n'aurait pas existé et sans qui les échanges n'auraient pas été aussi fructueux. 


\section{Références}

Best, J. R. et Miller, P. H. (2010). A developmental perspective on executive function. Child Development, 81(6), 1641-1660. https://doi.org/10.1111/j.1467-8624.2010.01499.x

Bunge, S. A., Dudukovic, N. M., Thomason, M. E., Vaidya, C. J. et Gabrieli, J. D. E. (2002). Immature frontal lobe contributions to cognitive control in children: Evidence from fMRI. Neuron, 33(2), 301-311. https://doi.org/10.1016/s0896-6273(01)00583-9

Cassotti, M. et Moutier, S. (2010). How to explain receptivity to conjunctionfallacy inhibition training: Evidence from the lowa Gambling Task. Brain and Cognition, 72(3), 378-384. https://doi.org/10.1016/j.bandc.2009.11.004

Chevalier, N. (2010). Les fonctions exécutives chez l'enfant: concepts et développement. Psychologie Canadienne, 51(3), 149-163. https://doi.org/10.1037/a0020031

Dempster, F. N. (1992). The rise and fall of the inhibitory mechanism: Toward a unified theory of cognitive development and aging. Developmental Review, 12(1), 45-75. https://doi.org/10.1016/0273-2297(92)90003-k

Dempster, F. N. et Corkill, A. (1999). Interference and inhibition in cognition and behavior: Unifying themes for educational psychology. Educational Psychology Review, 11(1), 1-88. https://doi.org/10.1023/a:1021992632168

Diamond, A., Barnett, W. S., Thomas, J. et Munro, S. (2007). The early years: Preschool program improves cognitive control. Science, 318(5855), 1387-1388. https://doi.org/10.1126/science. 1151148

Diamond, A. et Lee, K. (2011). Interventions shown to aid executive function development in children 4 to 12 years old. Science, 333(6045), 959964. https://doi.org/10.1126/science.1204529 
Fayol, M., Hupet, M. et Largy, P. (1999). The acquisition of subject-verb agreement in written French. From novices to experts' errors. Reading and Writing, 11(2), 153-174. https://doi.org/10.1023/a:1008038127807

Fernandez-Duque, D., Baird, J. et Posner M. (2000). Executive attention and metacognitive regulation. Consciousness and Cognition 9(2), 288-307. https://doi.org/10.1006/ccog.2000.0447

Fuson, K. C., Smith, S. T. et Cicero, A. M. L. (1997). Supporting Latino first graders' ten-structured thinking in urban classrooms. Journal for Research in Mathematics Education, 28(6), 738-766. https://doi.org/10.2307/749640

Gagné, P. P. et Ainsley, L. (2002). Cerveau... mode d'emploi. Module 1. Montréal : Éditions de la Chenelière.

Gagné, P. P. et Longpré, L. P. (2004). Apprendre... avec Réflecto. Module 3. Montréal : Éditions de la Chenelière.

Gagné, P. P. et Noreau, D. (2005). Le langage du temps. Module 2. Montréal : Éditions de la Chenelière.

Gaux, C. et Boujon, C. (2007). Développement du contrôle exécutif. Dans A. Blaye et $\mathrm{P}$. Lemaire (dir.), Psychologie du développement cognitif de l'enfant (p. 253-281). Bruxelles : De Boeck.

Goldin-Meadow, S. et Wagner, S. M. (2005). How our hands help us learn. Trends in Cognitive Sciences, 9(5), 234-241. https://doi.org/10.1016/j.tics.2005.03.006

Houdé, O. (2007). First insights on « neuropedagogy of reasoning ». Thinking and Reasoning, 13(2), 81-89. https://doi.org/10.1080/13546780500450599

Houdé, O. (2004/2011). La psychologie de l'enfant (Que sais-je?). Paris: PUF.

Houdé, O. (2000). Inhibition and cognitive development: object, number, categorization, and reasoning. Cognitive Development, 15(1), 63-73. https://doi.org/10.1016/s0885-2014(00)00015-0 
Houdé, O. et Guichard, E. (2001). Negative priming effect after inhibition of number/length interference in a Piaget-like task. Developmental Science, 4(1), 119-123. https://doi.org/10.1111/1467-7687.00156

Houdé, O. et Moutier, S. (1996). Deductive reasoning and experimental inhibition training: The case of the matching bias. Current Psychology of Cognition, 15(4), 409-434.

Houdé, O., Pineau, A., Leroux, G., Poirel, N., Perchey, G., Lanoë, C., ... et Mazoyer, B. (2011). Functional magnetic resonance imaging study of Piaget's conservation-of-number task in preschool and school-age children: A neo-Piagetian approach. Journal of Experimental Child Psychology, 110(3), 332-346. https://doi.org/10.1016/j.jecp.2011.04.008

Houdé, O., Zago, L., Mellet, E., Moutier, S., Pineau, A., Mazoyer, B. et Tzourio-Mazoyer, N. (2000). Shifting from the perceptual brain to the logical brain: the neural impact of cognitive inhibition training. Journal of Cognitive Neuroscience, 12(5), 721-728. https://doi.org/10.1162/089892900562525

Huizinga, M., Dolan, C. V. et van der Molen, M. W. (2006). Age-related change in executive function: Developmental trends and a latent variable analysis. Neuropsychologia, 44(11), 2017-2036. https://doi.org/10.1016/j.neuropsychologia.2006.01.010

Largy, P. (2001). La révision des accords nominal et verbal chez l'enfant. L'Année Psychologique, 101(2), 221-245. https://doi.org/10.3406/psy.2001.29554

Lubin, A., Poirel, N., Rossi, S., Lanoë, C., Pineau, A. et Houdé, O. (2010). Pedagogical effect of action on arithmetic performances in Wynn-like tasks solved by 2-year-olds. Experimental Psychology, 57(6), 405-411. https://doi.org/10.1027/1618-3169/a000049

Lubin, A., Poirel, N., Rossi, S., Pineau, A. et Houdé, O. (2009). Math in actions: Actor mode reveals the true arithmetic abilities of Frenchspeaking 2-year-olds in a magic task. Journal of Experimental Child Psychology, 103(3), 376-385. https://doi.org/10.1016/j.jecp.2009.02.002 
Masson, S., Potvin, P., Riopel, M. et Brault Foisy, L.-M. (2012). Identification des mécanismes cérébraux liés à l'expertise et retombées éducatives. Communication orale présentée au colloque Méthodologie de recherche en neuroéducation et retombées éducatives, dans le cadre du $17^{e}$ Congrès international de l'Association Mondiale des Sciences de l'Éducation. Reims (France), 3-8 Juin.

McDowd, J. M., Oseas-Kreger, D. M. et Filion, D. L. (1995). Inhibitory processes in cognition and aging. Dans F. N. Dempster et C. J. Brainerd (dir.), Interference and inhibition in cognition (p. 363-400). San Diego, CA: Academic Press. https://doi.org/10.1016/b978$\underline{012208930-5 / 50012-x}$

Moutier, S. (2000). Deductive reasoning and experimental matching-bias inhibition training in school children. Current Psychology of Cognition, 19(4), 429-452.

Moutier, S., Angeard, N. et Houde, O. (2002). Deductive reasoning and matching-bias inhibition training: Evidence from a debiasing paradigm. Thinking \& Reasoning, 8(3), 205-224. https://doi.org/10.1080/13546780244000033

Moutier, S. et Houdé, O. (2003). Judgement under uncertainty and conjunction fallacy inhibition training. Thinking \& Reasoning, 9(3), 185201. https://doi.org/10.1080/13546780343000213

Nunes, T., Bryant, P. et Bindman, M. (2006). The effects of learning to spell on children's awareness of morphology. Reading and Writing, 19(7), 767-787. https://doi.org/10.1007/s11145-006-9025-y

Romine, C. B. et Reynolds, C. R. (2005). A model of the development of frontal lobe functioning: Findings from a meta-analysis. Applied Neuropsychology, 12(4), 190-201. https://doi.org/10.1207/s15324826an1204 2 
Rossi, S., Leroux, G., Lubin, A., Lanoe, C., Poirel, N., Pineau, A. et Houdé, O. (2010). Imagerie du développement des enfants d'âge scolaire: Apports de la neuroimagerie à la compréhension du développement cognitif de l'enfant en lien avec les apprentissages scolaires. Applications en psychopédagogie. Communication orale dans le cadre de la $4^{\mathrm{e}}$ Édition des Entretiens Francophones de la Psychologie. Paris, France.

Rossi, S., Lubin, A., Pineau, A., Lanoë, C., Simon, G. et Houdé, O. (2012a). Une pédagogie du contrôle cognitif pour l'amélioration des capacités d'attention sélective: illustration chez l'enfant de 4-5 ans. Communication au colloque Méthodologie de recherche en neuroéducation et retombées éducatives, dans le cadre du $17^{\mathrm{e}}$ Congrès international de l'Association Mondiale des Sciences de l'Education. Reims, France. 3-8 Juin.

Rossi, S., Lubin, A., Pineau, A., Lanoë, C., Simon, G. et Houdé, O. (2011). Du laboratoire au terrain: regards croisés entre la recherche fondamentale et l'école sur l'importance du contrôle inhibiteur lors des premiers apprentissages scolaires. Communication orale dans le cadre du colloque OUtils pour la FORmation, l'Education et la Prévention. Nantes, France. 6-7 juin.

Rossi, S., Lubin, A., Lanoë, C. et Pineau A. (2012b). Une pédagogie du contrôle cognitif pour l'amélioration de l'attention à la consigne chez l'enfant de 4-5 ans. Neuroéducation, 1(1), 29-54.

https://doi.org/10.24046/neuroed.20120101.29

Shimamura, A. P. (2000). Toward a cognitive neuroscience of metacognition. Consciousness and Cognition, 9(2), 313-323. https://doi.org/10.1006/ccog.2000.0450

Siegler, R. S. (1999). Strategic development. Trends in Cognitive Sciences, 3(11), 430-435. https://doi.org/10.1016/s1364-6613(99)01372-8 
Totereau, C., Barrouillet, P. et Fayol, M. (1998). Overgeneralizations of number inflections in the learning of written French: The case of nouns and verbs. British Journal of Developmental Psychology, 16(4), 447464. https://doi.org/10.1111/j.2044-835x.1998.tb00764.x

Veenman, M., Van Hout-Wolters, B. et Afflerbach, P. (2006). Metacognition and learning: conceptual and methodological considerations. Metacognition Learning, 1(1), 3-14. https://doi.org/10.1007/s11409$\underline{\text { 006-6893-0 }}$

Walker, J. et Hauerwas, L. (2006). Development of phonological, morphological, and orthographic knowledge in young spellers: The case of inflected verbs. Reading and Writing, 19, 819-843.

https://doi.org/10.1007/s11145-006-9006-1 